Esta obra está sob o direito de Licença Creative Commons Atribuição 4.0 Internacional.

\title{
GAMETERAPIA COMO TECNOLOGIA ASSISTIVA: UMA REVISÃO SITEMÁTICA INTEGRATIVA
}

\author{
Sandra Maria Pontes ${ }^{1}$ \\ Andrea Marques Vanderlei Fregadolli ${ }^{2}$ \\ Bernard Pereira Almeida ${ }^{3}$
}

\section{RESUMO}

A introdução de recursos tecnológicos nos processos terapêuticos ocupacionais se apresenta como uma estratégia para atender às demandas dos indivíduos que exploram e acompanham as inovações tecnológicas, incluindo aqueles que apresentam alguma deficiência neuromotora. A pesquisa buscou analisar a produção científica sobre a Gameterapia como tecnologia assistiva no processo de reabilitação terapêutica, analisando produções científicas publicadas em 2019, nos sites periódicos da CAPES, da BVS. Scielo e ScienceDierct. Trata-se de uma revisão sistemática integrativa. Utilizando os descritores estruturados no DeCS e MeSH. O período de coleta dos dados ocorreu em junho/2019. Adotaram-se como critérios de inclusão artigos científicos e, como critérios de exclusão artigos que não contemplam a temática estudada. Espera-se que com novos estudos ocorra uma ampliação nessa área de intervenção fisioterapêutica favorecendo tratamentos cada vez mais eficiente a todos os pacientes.

PALAVRAS-CHAVE: Gameterapia. Tecnologia Assistiva. Reabilitação Terapêutica.

\footnotetext{
${ }^{1}$ Sandrinha.2005@ hotmail.com

2 deadoutorado@hotmail.com

3 bernardadv@hotmail.com
} 


\section{INTRODUÇÃO}

A introdução de recursos tecnológicos nos processos terapêuticos ocupacionais se apresenta como uma estratégia para atender às demandas dos indivíduos que exploram e acompanham as inovações tecnológicas, incluindo aqueles que apresentam alguma deficiência neuromotora. Desse modo, evidencia-se a intervenção no escopo da terapia ocupacional realçando a sua influência na melhora do desempenho em uma atividade significativa. Destaca -se a ação do paciente em jogar videogame e, concomitantemente, as consequências adquiridas no tocante a melhora de habilidades cognitivas subjacentes como também um avanço nas outras atividades cotidianas (DIA et al., 2019).

Os autores acima supracitados, afirmam que as sessões de gameterapia apresentam uma forte contribuição no tocante à aquisição e o aprimoramento dessas habilidades cognitivas, como também um avanço significativo nos aspectos voltados para a incorporação de um recurso que estimula a motivação e a participação no processo de terapêutico, visto que esta é considerada uma atividade de lazer para o paciente. Portanto, a utilização de uma abordagem diferenciada da terapia convencional pode ter permitido ao indivíduo a atribuição de um novo sentido ao seu processo terapêutico.

\section{MÉTODOLOGIA}

Trata-se de uma revisão bibliográfica, do tipo sistemática integrativa, de natureza qualitativa, que seguiu seis etapas (ver Quadro 1): definição do tema; seleção da pergunta norteadora e escolha da estratégia de busca; descritores e bases de dados mais eficazes no levantamento das publicações; categorização dos estudos; a avaliação crítica dos estudos selecionados; análise; interpretação e discussão dos resultados e a apresentação da revisão em formato de artigo o qual contempla as propostas para estudos futuros. 


\section{RESULTADOS}

Tabela 1- Corresponde ao quantitativo das varreduras realizadas em cinco bases de buscas. Foram detectadas 1.911,00 publicações científicas nos bancos de dados, das quais 725 eram artigos científicos disponíveis após o uso dos filtros, desses foram feitos 125 downloads. Entretanto, obedeceram aos critérios de inclusão 7 artigos científicos, sendo submetidos às etapas da revisão integrativa.

Tabela 1 - String de busca e bibliotecas virtuais consultadas.

\begin{tabular}{|c|c|c|c|c|c|}
\hline $\begin{array}{l}\text { Descritores/ } \\
\text { String de } \\
\text { busca }\end{array}$ & $\begin{array}{c}\text { Bases de } \\
\text { dados }\end{array}$ & $\begin{array}{c}\text { Total de } \\
\text { publicações } \\
\text { sem o filtro } \\
\text { "Assunto } \\
\text { principal" }\end{array}$ & $\begin{array}{l}\text { Textos } \\
\text { completos } \\
\text { após aplicar } \\
\text { filtros }\end{array}$ & $\begin{array}{l}\text { Downloads } \\
\text { de textos } \\
\text { completos }\end{array}$ & $\begin{array}{c}\text { Textos aproveitados na } \\
\text { Revisão Sistemática } \\
\text { Integrativa }\end{array}$ \\
\hline \multirow{5}{*}{$\begin{array}{c}1^{a} \text { String } \\
\text { Gameterapia }\end{array}$} & Scielo & 2 & 2 & 2 & - \\
\hline & $\begin{array}{l}\text { Periódico } \\
\text { da CAPES }\end{array}$ & 2 & 2 & 2 & 1 \\
\hline & $B V S$ & 5 & 5 & 5 & 1 \\
\hline & WILEY & 1 & 1 & 1 & - \\
\hline & $\begin{array}{c}\text { ScienceDie } \\
\text { rect }\end{array}$ & ----- & ------ & ----- & ----- \\
\hline \multirow{4}{*}{$\begin{array}{c}2^{a} \text { string } \\
\text { therapy AND } \\
\text { videogame }\end{array}$} & Scielo & 10 & 7 & 6 & --- \\
\hline & Wiley & 421 & 187 & 15 & 2 \\
\hline & $\begin{array}{c}\text { ScienceDir } \\
\text { ect }\end{array}$ & 567 & 61 & 55 & 2 \\
\hline & $\begin{array}{l}\text { Periódicos } \\
\text { da CAPES }\end{array}$ & 904 & 460 & 41 & 1 \\
\hline Total & & $1.911,00$ & 725 & 127 & 7 \\
\hline
\end{tabular}

FONTE: elaborada pelos autores.

Dos 10 artigos (Quadro 4) analisados, 3 eram estudos secundários(revisão) e 4 eram estudos primários (2 relatos de experiência, 1 estudos de caso, e 1 relato de experiência). Quanto ao ano de publicação, foram selecionados apenas os publicados no de 2019. Os artigos estavam escritos em inglês 
Quadro 2 - Descrição dos documentos (artigos) de acordo com os critérios de inclusão. $1^{a}$ string - Gameterapia

\begin{tabular}{|c|c|c|c|c|c|}
\hline BASE & AUTOR(A) & TEMA & ANO & $\begin{array}{c}\text { OBJETIVO } \\
\text { DO } \\
\text { ESTUDO }\end{array}$ & CONCLUSÃO DO ESTUDO \\
\hline BVS & Dia et all & $\begin{array}{c}\text { Contribuições } \\
\text { da gameterapia } \\
\text { para as } \\
\text { habilidades } \\
\text { cognitivas de } \\
\text { um } \\
\text { adolescente } \\
\text { com paralisia } \\
\text { cerebral }^{1}\end{array}$ & 2019 & $\begin{array}{c}\text { Analisar as } \\
\text { contribuições } \\
\text { da } \\
\text { gameterapia } \\
\text { para as } \\
\text { habilidades } \\
\text { cognitivas de } \\
\text { um } \\
\text { adolescente } \\
\text { com paralisia } \\
\text { cerebral. }\end{array}$ & $\begin{array}{l}\text { A introdução de recursos tecnológicos } \\
\text { nos processos terapêuticos ocupacionais } \\
\text { se apresenta como uma estratégia para } \\
\text { atender às demandas dos sujeitos que } \\
\text { exploram e acompanham as inovações } \\
\text { tecnológicas, incluindo sujeitos que } \\
\text { apresentam alguma deficiência } \\
\text { neuromotora. Desse modo, a } \\
\text { intervenção no escopo da terapia } \\
\text { ocupacional visa a melhora do } \\
\text { desempenho em uma atividade } \\
\text { significativa (jogar videogame) para o } \\
\text { sujeito e, concomitantemente, a } \\
\text { melhora de habilidades cognitivas } \\
\text { subjacentes a outras atividades } \\
\text { cotidianas. Desse modo, as sessões de } \\
\text { gameterapia podem ter contribuído para } \\
\text { a aquisição e o aprimoramento de } \\
\text { habilidades cognitivas pelo sujeito da } \\
\text { pesquisa, levando em consideração a } \\
\text { incorporação de um recurso que } \\
\text { estimule a motivação e a participação } \\
\text { no processo de terapêutico, visto que } \\
\text { esta é considerada uma atividade de } \\
\text { lazer para o sujeito. Portanto, a } \\
\text { utilização de uma abordagem } \\
\text { diferenciada da terapia convencional } \\
\text { pode ter permitido ao indivíduo a } \\
\text { atribuição de um novo sentido ao seu } \\
\text { processo terapêutico. } \\
\text { Verificou-se assim, através da pesquisa, } \\
\text { que a gameterapia pode se apresentar } \\
\text { como estratégia terapêutica } \\
\text { ocupacional, por possibilitar melhora } \\
\text { nas habilidades cognitivas e no } \\
\text { engajamento do paciente no tratamento, } \\
\text { sendo aliada a outros métodos de } \\
\text { tratamento de indivíduos com PC. } \\
\text { Nesta perspectiva, ressalta-se a } \\
\text { importância da realização de estudos na } \\
\text { área de gameterapia com enfoque em } \\
\text { habilidades cognitivas, visto que a } \\
\text { proporção de estudos com enfoque } \\
\text { nestas habilidades é muito menor } \\
\text { quando comparada a estudos que } \\
\text { investigam a utilização de videogames } \\
\text { para a estimulação motora, embora, } \\
\text { conforme mostrado neste estudo, este } \\
\text { recurso possa contribuir para a } \\
\text { viabilização da aquisição e/ou } \\
\text { aprimoramento de habilidades } \\
\text { cognitivas. }\end{array}$ \\
\hline
\end{tabular}




\begin{tabular}{|l|l|l|l|}
\hline & & & $\begin{array}{c}\text { Ressalta-se que, nesta pesquisa, o uso } \\
\text { do método de estudo de caso configura } \\
\text { uma limitação quanto à validade } \\
\text { externa e ao controle de variáveis, o que } \\
\text { limita a generalização dos resultados. } \\
\text { Portanto, sugere-se a utilização de } \\
\text { grupos-controle em pesquisas futuras. }\end{array}$ \\
\hline
\end{tabular}

$2^{\text {a }}$ String - therapy AND videogame

\begin{tabular}{|c|c|c|c|c|c|}
\hline BASES & CITAÇÃO & TEMA & ANO & $\begin{array}{l}\text { OBJETIVO DO } \\
\text { ESTUDO }\end{array}$ & CONCLUSÃO DO ESTUDO \\
\hline WILEY & Woodberry et all & $\begin{array}{l}\text { Computer- } \\
\text { aided learning } \\
\text { for managing } \\
\text { stress: A } \\
\text { feasibility trial } \\
\text { with clinical } \\
\text { high risk } \\
\text { adolescents } \\
\text { and young } \\
\text { adults }\end{array}$ & 2019 & $\begin{array}{l}\text { Testar a viabilidade } \\
\text { de integrar o } \\
\text { biofeedback e o jogo } \\
\text { cooperativo de } \\
\text { videogame na terapia } \\
\text { familiar como um } \\
\text { meio de envolver e } \\
\text { aumentar a resiliência } \\
\text { dessa população. }\end{array}$ & $\begin{array}{l}\text { Os videogames multiusuário podem } \\
\text { ter um papel a desempenhar no } \\
\text { envolvimento de jovens em risco de } \\
\text { psicose na terapia e na redução do } \\
\text { estresse e dos fatores de risco } \\
\text { familiares. Uma série de } \\
\text { desenvolvimentos importantes são } \\
\text { necessários para aumentar o apelo, a } \\
\text { eficácia e a praticidade dessa } \\
\text { abordagem. }\end{array}$ \\
\hline WILEY & Boon et all & $\begin{array}{l}\text { Treatment and } \\
\text { compliance } \\
\text { with virtual } \\
\text { reality and } \\
\text { anaglyph- } \\
\text { based training } \\
\text { programs for } \\
\text { convergence } \\
\text { insufficiency } \\
\end{array}$ & 2019 & $\begin{array}{l}\text { avaliar a viabilidade } \\
\text { do uso de gamificação } \\
\text { do treinamento da } \\
\text { visão para: (a) tratar a } \\
\text { insuficiência de } \\
\text { convergência; e (b) } \\
\text { melhorar a adesão ao } \\
\text { tratamento em } \\
\text { comparação com um } \\
\text { tratamento } \\
\text { convencional durante } \\
\text { um período de } \\
\text { tratamento de seis } \\
\text { semanas. }\end{array}$ & $\begin{array}{l}\text { A gamificação do treinamento de } \\
\text { visão em um ambiente de realidade } \\
\text { virtual é viável e associada a maior } \\
\text { conformidade, portanto, pode ser } \\
\text { uma estratégia útil para tratar a } \\
\text { insuficiência de convergência. }\end{array}$ \\
\hline $\begin{array}{c}\text { Science } \\
\text { direct }\end{array}$ & $\begin{array}{c}\text { Filipa Ferreira- } \\
\text { Britoa, } *, \\
\text { Mónica Fialhoa, } \\
\text { Ana Virgolinoa } \\
\text {, Inês Nevesa , } \\
\text { Ana Cristina } \\
\text { Mirandaa,b, } \\
\text { Nuno Sousa- } \\
\text { Santosa,c, Cátia } \\
\text { Caneirasa,d, } \\
\text { Luís Carriçoe,f, } \\
\text { Ana } \\
\text { Verdelhoa,g,h, } \\
\text { Osvaldo } \\
\text { Santosa }\end{array}$ & $\begin{array}{l}\text { Game-based } \\
\text { interventions } \\
\text { for } \\
\text { neuropsycholo } \\
\text { gical } \\
\text { assessment, } \\
\text { training and } \\
\text { rehabilitation: } \\
\text { Which game- } \\
\text { elements to } \\
\text { use? A } \\
\text { systematic } \\
\text { review }\end{array}$ & 2019 & $\begin{array}{c}\text { Este estudo teve como } \\
\text { objetivo identificar } \\
\text { GE aplicado em GBI } \\
\text { para avaliação } \\
\text { cognitiva, treinamento } \\
\text { ou reabilitação. }\end{array}$ & $\begin{array}{l}\text { No entanto, o desenvolvimento de } \\
\text { GBI que são baseados na } \\
\text { implementação de números sistemas } \\
\text { de feedback podem comprometer o } \\
\text { objetivo principal com o qual este } \\
\text { tipo de intervenções tem sido usado: } \\
\text { promoção da motivação intrínseca } \\
\text { em direção a objetivos de longo } \\
\text { prazo. Além disso, a falta de } \\
\text { qualquer outro associação entre GE } \\
\text { usado e os resultados cognitivos } \\
\text { direcionados enfatiza a necessidade } \\
\text { de definir um quadro teórico que } \\
\text { apoia a seleção estratégica de GE de } \\
\text { acordo com o paciente / patologia } \\
\text { características, bem como de acordo } \\
\text { com as características das } \\
\text { construções cognitivas que são } \\
\text { direcionados por intervenções } \\
\text { específicas baseadas em jogos. }\end{array}$ \\
\hline
\end{tabular}




\begin{tabular}{|c|c|c|c|c|c|}
\hline $\begin{array}{l}\text { Scienced } \\
\text { irect }\end{array}$ & $\begin{array}{c}\text { Eatedal } \\
\text { Alabdulakareem } \\
\text {, Mona } \\
\text { Jamjoom* }\end{array}$ & $\begin{array}{c}\text { Computer- } \\
\text { assisted } \\
\text { learning for } \\
\text { improving } \\
\text { ADHD } \\
\text { individuals' } \\
\text { executive } \\
\text { functions } \\
\text { through } \\
\text { gamified } \\
\text { interventions: } \\
\text { A review }\end{array}$ & 2019 & $\begin{array}{l}\text { Este artigo investigou } \\
\text { os efeitos de } \\
\text { intervenções } \\
\text { gamificadas no } \\
\text { desempenho de } \\
\text { indivíduos com } \\
\text { TDAH, revisando } \\
\text { jogos descritos na } \\
\text { literatura que foram } \\
\text { desenvolvidos } \\
\text { principalmente para } \\
\text { melhorar os FEs de } \\
\text { indivíduos com } \\
\text { TDAH. }\end{array}$ & $\begin{array}{l}\text { Com as inovações tecnológicas, as } \\
\text { contribuições do CAL aumentaram } \\
\text { imensamente, especialmente no que } \\
\text { diz respeito a ajudar pessoas com } \\
\text { necessidades especiais. Uma } \\
\text { estratégia de CAL promissora é a } \\
\text { intervenção gamificada (ou seja, } \\
\text { SGs), que pode ser usada como uma } \\
\text { intervenção de treinamento especial } \\
\text { para pessoas diagnosticadas com } \\
\text { TDAH. Este artigo analisou os } \\
\text { estudos existentes de SG com TDAH } \\
\text { e explorou os efeitos dos SGs no } \\
\text { desempenho de indivíduos com } \\
\text { TDAH. A maioria dos estudos } \\
\text { relatou melhora no engajamento e } \\
\text { motivação de indivíduos com } \\
\text { TDAH, juntamente com outras } \\
\text { habilidades que os jogos visam. A } \\
\text { intervenção gamificada é uma } \\
\text { técnica de aprendizagem promissora } \\
\text { que melhora os FEs de indivíduos } \\
\text { com TDAH; ele pode ser usado } \\
\text { como um suplemento de tratamento } \\
\text { não médico ou alternativa. } \\
\text { O documento também esclareceu } \\
\text { algumas das limitações dos SGs e } \\
\text { sugeriu possíveis soluções para elas } \\
\text { que os inventores do jogo poderiam } \\
\text { considerar no futuro. Além disso, } \\
\text { descobriu que integrar SGs com TEL } \\
\text { poderia ajudar pessoas que sofrem } \\
\text { de TDAH, mantendo sua motivação } \\
\text { e engajamento dentro do jogo por } \\
\text { meio de dispositivos interativos } \\
\text { avançados. No entanto, isso precisa } \\
\text { ser equilibrado, já que usar SGs por } \\
\text { longos períodos de tempo pode levar } \\
\text { ao vício em jogos, entre outros } \\
\text { problemas. Portanto, educadores, } \\
\text { designers, programadores, terapeutas } \\
\text { e outras partes interessadas devem } \\
\text { estar envolvidos na criação de SGs } \\
\text { para maximizar seus benefícios e } \\
\text { controlar suas desvantagens. No } \\
\text { futuro, os presentes pesquisadores } \\
\text { pretendem desenvolver um GS que } \\
\text { atenda a algumas das recomendações } \\
\text { aqui incluídas. }\end{array}$ \\
\hline
\end{tabular}




\begin{tabular}{|c|c|c|c|c|c|}
\hline $\begin{array}{l}\text { Periódic } \\
\text { o da } \\
\text { CAPES }\end{array}$ & Tao et all & $\begin{array}{l}\text { Self-directed } \\
\text { usage of an in- } \\
\text { home } \\
\text { exergame after } \\
\text { a supervised } \\
\text { telerehabilitati } \\
\text { on training } \\
\text { program for } \\
\text { older adults } \\
\text { with lower- } \\
\text { limb }\end{array}$ & 2019 & $\begin{array}{l}\text { Examine o uso de um } \\
\text { exergame em casa, em } \\
\text { comparação com o } \\
\text { controle, não } \\
\text { supervisionado após o } \\
\text { treinamento } \\
\text { supervisionado por } \\
\text { idosos com amputação } \\
\text { de membros } \\
\text { inferiores. }\end{array}$ & $\begin{array}{l}\text { Comparado a uma fase de } \\
\text { treinamento supervisionado por um } \\
\text { médico, os participantes dos grupos } \\
\text { de jogos de exercícios WiiNWalk e } \\
\text { BBA sem exercícios usaram seus } \\
\text { dispositivos de treinamento com } \\
\text { menos frequência na fase não } \\
\text { estruturada não supervisionada. } \\
\text { Enquanto os participantes do grupo } \\
\text { experimental demonstraram tempos } \\
\text { de exercício consistentes entre as } \\
\text { fases supervisionadas e não } \\
\text { supervisionadas, a inclusão de } \\
\text { elementos de videogame por si só } \\
\text { pode ser insuficiente para motivar } \\
\text { regimes autodirecionados de } \\
\text { exercícios em ambiente doméstico } \\
\text { não supervisionado. Portanto, } \\
\text { projetos futuros de intervenções de } \\
\text { exergame domiciliares podem se } \\
\text { beneficiar pela inclusão de dados } \\
\text { clínicos sustentáveis ou } \\
\text { envolvimento social. }\end{array}$ \\
\hline $\begin{array}{l}\text { Periódic } \\
\text { o da } \\
\text { CAPES }\end{array}$ & Agundez at al & $\begin{array}{c}\text { Parkinson's } \\
\text { Disease with } \\
\text { Exergames: A } \\
\text { Systematic } \\
\text { Review }\end{array}$ & 2019 & $\begin{array}{l}\text { O objetivo desta } \\
\text { contribuição é reunir e } \\
\text { analisar criticamente } \\
\text { as evidências recentes } \\
\text { sobre o potencial do } \\
\text { exergame para a } \\
\text { reabilitação da doença } \\
\text { de Parkinson (DP) e } \\
\text { fornecer uma análise } \\
\text { atualizada do estado } \\
\text { atual dos estudos } \\
\text { sobre terapia baseada } \\
\text { em exergame em } \\
\text { pacientes com DP. }\end{array}$ & $\begin{array}{l}\text { Em nossa opinião, a principal } \\
\text { conclusãa deste estudo é que a } \\
\text { reabilitação com exergame DP é de } \\
\text { fato segura, viável e eficaz e, em } \\
\text { alguns casos [37, 38, 40, 42], ainda } \\
\text { mais eficaz do que a reabilitação } \\
\text { tradicional e exercícios regulares. Os } \\
\text { Exergames também oferecem a } \\
\text { possibilidade de um cenário de } \\
\text { reabilitação domiciliar e } \\
\text { monitoramento remoto, que são } \\
\text { limitações dos métodos tradicionais } \\
\text { de reabilitação. Há evidências } \\
\text { contraditórias sobre qual plataforma } \\
\text { de exergame tem melhor } \\
\text { desempenho neste ponto. } \\
\text { Com relação aos trabalhos futuros, } \\
\text { além de fornecer mais ensaios } \\
\text { clínicos de alta qualidade para } \\
\text { confirmar as hipóteses até agora } \\
\text { apresentadas, os estudos devem se } \\
\text { concentrar na adaptação das terapias } \\
\text { existentes aos cenários domésticos, } \\
\text { acrescentando outros procedimentos } \\
\text { de coleta de dados, como } \\
\text { monitoramento de tremores nas } \\
\text { mãos, talvez com um sensor de dedo } \\
\text { [82]. Adicional } \\
\text { variáveis como disfonia [83] e } \\
\text { variabilidade da frequência cardíaca } \\
\text { [84] também podem ser de interesse. } \\
\text { Avaliar a viabilidade de expandir a } \\
\text { terapia PD Exergame para pacientes } \\
\text { com PD-MCI e PDD e incluir novas } \\
\text { técnicas de interação, como } \\
\text { treinamento motor fino e controle da } \\
\text { fala também são pontos de interesse. } \\
\text { Finalmente, os programas devem ser } \\
\text { adaptados às características do } \\
\text { paciente, tanto físicas quanto } \\
\text { cognitivas. }\end{array}$ \\
\hline
\end{tabular}

FONTE: Plataforma online das bases científicas, 2019. 
O escopo textual foi analisado por meio da frequência de palavras, que originou a nuvem de palavras (Figura 1) criada na plataforma online WordArt. Esta ferramenta agrupa, alinha e destaca graficamente as palavras-chaves mais frequentes.

Tabela 2. Frequência das palavras extraídas das conclusões dos artigos baixados das bases científicas.

\begin{tabular}{|c|c|c|}
\hline PALAVRAS & FREQUÊNCIA & CATEGORIAS \\
\hline Gameterapia & 3 & \multirow{3}{*}{$\begin{array}{c}\text { Gameterapia } \\
\text { como } \\
\text { estratégica } \\
\text { terapêutica. }\end{array}$} \\
\hline Estratégica & 1 & \\
\hline Terapêutica & 6 & \\
\hline Intervenção & 12 & \multirow{2}{*}{$\begin{array}{l}\text { Estudo de } \\
\text { intervenção } \\
\text { gamificada }\end{array}$} \\
\hline Gamificada & 2 & \\
\hline
\end{tabular}

FONTE: elaborada pelos autores

Figura 1 - Nuvem de palavras:

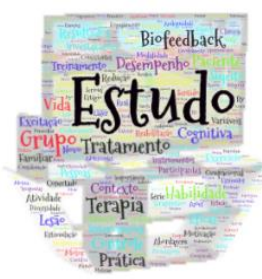

FONTE: elaborada pelo autor.

Mediante a Figura 1, foi possível constatar que as palavras em evidência na nuvem pertencem as categorias desenvolvidas a partir da análise de conteúdo de Bardin. Toas as categorias advêm da sua frequência (Tabela 2), que diz respeito ao seu quadro referencial. De acordo com o objetivo deste estudo, preferiu-se por caracterizar as palavras que apresentaram maior frequência no escopo do texto e, a partir dos seus conteúdos semânticos, tinham uma magnitude expressiva no contexto das concepções alinhadas a temática da gameterapia como tecnologia assistiva, como destacado na Figura 1. Tabela 2 - Frequência das palavras presentes nos artigos publicados pelos internautas nas plataformas de bases científicas (BVS, WYLEI, SCIENCEDIRECT, PERIÓDICO DA CAPES, SCIELO).

\section{DISCUSSÃO}

No campo da recuperação fisioterapêutica há diversas estratégias ligadas a esse processo, no entanto a gameterapia traz no seu escopo a dinâmica lúdica como carro chefe motivacional para que os pacientes interajam com mais prazer e realizem os exercícios propostos. Contudo, faz-se necessãrio destacar que a inclusão de elementos de videogame por si só pode ser insuficiente para motivar regimes autodirecionados de exercícios em ambiente doméstico não supervisionado (Tao et all, 2019).Seguem abaixo, as categorias temáticas elaboradas a partir da revisão sistemática integrativa:

\section{GAMETERAPIA ESTRATÉGIA TERAPÊUTICA OCUPACIOANL}


A intervenção no escopo da terapia ocupacional visa a melhora do desempenho em uma atividade significativa (jogar videogame) para o paciente e, concomitantemente, a melhora de habilidades cognitivas subjacentes a outras atividades cotidiana, fato crucial para apreensão influenciadora da gameterapia (DIA et all, 2019).

A gameterapia, assim foi constatada após alguns estudos, pode se apresentar como estratégia terapêutica ocupacional, devido a mesma sinalizar possibilidades de um melhoras nas habilidades cognitivas e no engajamento do paciente no tratamento, sendo aliada a outros métodos de tratamento de indivíduos com PC DIAS et all, 2019. Vale ressaltar que, há uma série de desenvolvimentos importantes considerados necessários para aumentar o apelo, a eficácia e a praticidade dessa abordagem (WOODBERRY et all, 2019).

No entanto, alguns estudos que são baseados na implementação de feedback podem comprometer o objetivo principal com o qual este tipo de intervenções tem sido usado: promoção da motivação intrínseca em direção a objetivos de longo prazo. Resultados cognitivos direcionados são enfáticos no tocante à necessidade de definir um quadro teórico que apoia a seleção estratégica para o tratamento específico que deve está de acordo com o paciente / patologia características, bem como de acordo com as características das construções cognitivas que são direcionados por intervenções específicas baseadas em jogos( BRITOA et all, 2019).

Nesse sentido, a inclusão de elementos de videogame por si só pode ser insuficiente para motivar regimes autodirecionados de exercícios em ambiente doméstico não supervisionado. Portanto, projetos futuros de intervenções de exergame domiciliares podem se beneficiar pela inclusão de dados clínicos sustentáveis ou envolvimento social(TAO et all, 2019).

Por fim, vale realçar essa possibilidade de um cenário de reabilitação domiciliar e monitoramento remoto, que são limitações dos métodos tradicionais de reabilitação. Com relação aos trabalhos futuros, além de fornecer mais ensaios clínicos de alta qualidade para confirmar as hipóteses até agora apresentadas, os estudos devem se concentrar na adaptação das terapias existentes aos cenários domésticos, acrescentando outros procedimentos de coleta de dados. Avaliar a viabilidade de expandir a terapia e incluir novas técnicas de interação, como treinamento motor fino e controle da fala também são pontos de interesse. Finalmente, os programas devem ser adaptados às características do paciente, tanto físicas quanto cognitivas ( AGUNDEZ et all, 2019) 


\section{ESTUDO DE INTERVENÇÃO GAMIFICADA}

Nesta perspectiva, ressalta-se a importância da realização de estudos na área de gameterapia com enfoque em habilidades cognitivas, visto que a proporção de estudos com enfoque nestas habilidades é muito menor quando comparada a estudos que investigam a utilização de videogames para a estimulação motora, embora, conforme mostrado neste estudo, este recurso possa contribuir para a viabilização da aquisição e/ou aprimoramento de habilidades cognitivas (DIA et all, 2019).

Trazendo como exemplo, a gamificação do treinamento de visão em um ambiente de realidade virtual é viável e associada a maior conformidade, portanto, pode ser uma estratégia útil para tratar a insuficiência de convergência (BOON et all, 2019).
Com as inovações tecnológicas, as contribuições de caráter inclusivo aumentaram imensamente, especialmente no que diz respeito a ajudar pessoas com necessidades especiais. Nesse contexto, evidencia-se, como sendo uma estratégia promissora é a intervenção gamificada (ou seja, SGs), que pode ser usada como uma intervenção de treinamento especial para pessoas diagnosticadas com TDAH. Uma parte considerável dos estudos relatou melhora no engajamento e motivação de indivíduos com TDAH, juntamente com outras habilidades que os jogos visam. Assim, desenha-se a intervenção gamificada evidenciando-a como uma técnica de aprendizagem promissora que melhora o quadro dos pacientes que , nesse caso apresentam TDAH; ele pode ser usado como um suplemento de tratamento não médico ou alternativa (ALABDULAKAREEM, JAMJOOM, 2019) 
Ainda, atrelado a esse pensamento, descobriu-se que promover a intregação desse pacientes nesse tratamento, poderia ajudar outras pessoas que sofrem de TDAH, mantendo sua motivação e engajamento dentro do jogo por meio de dispositivos interativos avançados. No entanto, isso precisa ser equilibrado, devido ao perído longo de uso corre o risco de levar ao vício em jogos, entre outros problemas. Portanto, educadores, designers, programadores, terapeutas $\mathrm{e}$ outras partes interessadas devem estar envolvidos na criação de possibilidades para maximizar seus benefícios e controlar suas desvantagens. No futuro, os presentes pesquisadores pretendem desenvolver uma intervenção gamificada que atenda a algumas das recomendações aqui incluídas (ALABDULAKAREEM, JAMJOOM, 2019)

\section{CONCLUSÃO}

O presente estudo sinaliza para uma intervenção gamificada com possibilidades promissoras no tocante a recuperação do paciente em tratamento fisioterapêutico. Elementos como ludicidade, motivação, são alguns dos benefícios que essa estratégia terapêutica carrega no seu escopo tornando-a irreverente nesse contexto.

Com possibilidades de ser realizada no ambiente familiar, a torna ainda mais atrativa haja vista que o seio familiar é assim considerado um elemento chave na recuperação acelerada do paciente.

Contudo, os estudiosos revelam a necessidade de novas pesquisas nessa esfera de tratamento baseado em videogame, ou seja, o uso dessa estratégia chamada de Gameterapia requer de fato um apanhado mais expressivo para assim consubstanciar os resultados encontrados e poder referenciá-los nas práticas futuras.

\section{REFERÊNCIAS}

AGUNDEZ, A.G.; FOLKERTS, K.A.;

KONRAD, R.; CASERMAN, P.; TREGEL, T.; GOOSSES, M.; GOBEL, S.; KALBE, E.

Parkinson's Disease with Exergames: A

Systematic Review, 2019.

ALABDULAKAREEM, E.; JAMJOOM, M. Computer-assisted learning for improving ADHD individuals' executive functions through gamified interventions: A review, 2019.

ASPER, L. J.; ALAGIAH, P.; BOON, Y.M.; CHIK, P.; Treatment and compliance with virtual reality and anaglyph-based training programs for convergence insufficiency, 2019.

BRITOA, F.If.; FIALHOA, M.; VIRGOLINOA, A.; NEVEA, I. A.; Gamebased interventions for neuropsychological assessment, training 
and rehabilitation: Which game-elements

to use? A systematic review, 2019.

DIAS, S.T.; CONCEIÇÃO, K. F.;

OLIVEIRA, O. A. I.; SILVA, M. L.

R.Contribuições da gameterapia para as

habilidades cognitivas de um adolescente

com paralisia cerebral1, 2019.

TAO, G.; MILLER, W.C.; ENG, J.J.;

LINDSTROM, H.; IMAM, B.; Self-

directed usage of an in-home exergame

after a supervised telerehabilitation

training program for older adults with

lower-limb, 2019.

WOODBERRY, K. A.; CHOKRAN . C.;

JOHNSON, K. A.; NUECHTERLEIN, H.

K.; MIKLOWITZ, J. D.; FARAONE, S. V.;

SEIDMAN, L.J.; Computer-aided

learning for managing stress: A

feasibility trial with clinical high-risk

adolescents and young adults, 2019. 